\title{
Novel Cross-Type Network for Wide-Tuning-Range Reconfigurable Multiband Antennas
}

\author{
Chieh-Sen Lee, Chi-Lin Tsai, and Chin-Lung Yang \\ Department of Electrical Engineering, National Cheng Kung University, Tainan 70101, Taiwan \\ Correspondence should be addressed to Chin-Lung Yang; cyang@mail.ncku.edu.tw
}

Received 1 April 2014; Accepted 11 June 2014; Published 26 August 2014

Academic Editor: Massimiliano Simeoni

Copyright ( 2014 Chieh-Sen Lee et al. This is an open access article distributed under the Creative Commons Attribution License, which permits unrestricted use, distribution, and reproduction in any medium, provided the original work is properly cited.

\begin{abstract}
This paper presents a cross-type network design with a novel reconfigurable functionality to realize a tunable multiband antenna. By attaching a reconfigurable network at the feeding port of a broadband antenna, multi-input impedance adjustment enables the production of multimatching operating bands. Each band can be independently controlled by a single component with a considerably wide tuning range and high selectivity. The experiments in this study involved using an ultra-wideband (UWB) antenna connected to the proposed cross-type network. The tunable antenna operates in a dual band of $f_{L}$ ( 1.39 to $2.34 \mathrm{GHz}$ ) and $f_{H}(2.1$ to $3.6 \mathrm{GHz})$ with tunable frequency ratios of $168 \%$ and $132 \%$, respectively. The average bandwidths at $f_{L}$ and $f_{H}$ are approximately $50 \mathrm{MHz}$ and $148 \mathrm{MHz}$, respectively, implying narrowband operation. The measured radiation pattern revealed that the tunable antenna exhibits a nearly omnidirectional radiation pattern at both 1.8 and $3.5 \mathrm{GHz}$. The network circuit architecture can be extended to the multiband function type by adopting this matching approach. The amount of shunt matches determines the number of operation bands.
\end{abstract}

\section{Introduction}

Because of the fast development of wireless communication technologies, multifunctional platforms and their quality of service have become a research focus [1]. Wireless frontend antennas with reconfigurable functions can improve the flexibility of channel reuse and capacity and also reduce the noise and the mutual interference between adjacent channels [2]. Frequency-reconfigurable antennas have been explored as an alternative to multiband antennas [3].

Numerous studies have analyzed reconfigurable and multiband structures, such as the microstrip patch antenna [4-7], PIFA [8, 9], and slot antenna [10-12]. Reconfigurable antennas can manipulate the current distribution of the structure and vary resonance frequencies. Switches and varactors, such as radio-frequency microelectromechanical systems and p-i-n diodes, have also been studied and directly incorporated into antenna structures to form frequency-reconfigurable antennas. Frequency tuning can also be achieved using tunable filters in a filter-based reconfiguration. A tunable filter embedded in an antenna can be also regarded as a tunable antenna [13-16]. The use of reconfigurable antennas has always been hindered by the need for a large number of switches, complex dc wiring, and the limitation of serving one frequency band at a time [2].

This paper introduces a novel and complete method for the design and full characterization of a reconfigurable multiband structure with a cross-type front-end network circuit to achieve a tunable mechanism $[17,18]$. The proposed cross-type network is based on a T-type impedance-matching network. As indicated in Figure 1, this front-end network can be embedded in any broadband system or antenna and expanded from a single tunable band to a dual-band network. The cross-type network exhibits an additional frequencyselection ability. The functionality of network to create a tunable antenna was verified in this study. The design flow of the tunable network can be analyzed on an $L C$ network, which provides powerful and quick electronic design automation (EDA) tools such as SPICE and ADS. This tunable network features controllable operation band numbers, continuous 


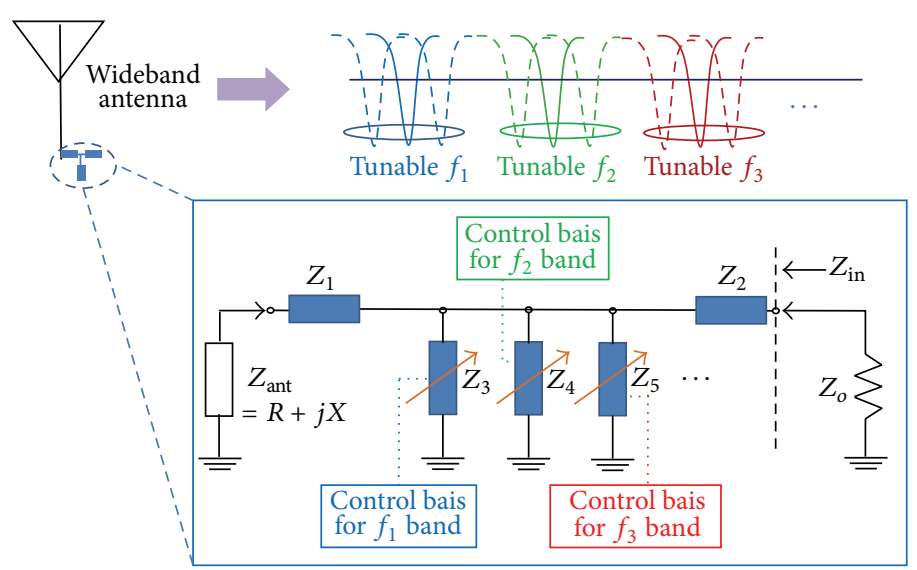

FIGURE 1: Tunable multiband system using novel tunable cross-type network.

tuning with a single corresponding control, independent control with a wide range in each band, high selectivity, and easy minimization after integration.

\section{A Tunable Cross-Type Network for Multiband Operation Design}

According to the matching theorem, one network reactance corresponds to one matching operating frequency. Dualband operation uses the unique characteristics of a crosstype matching network to simultaneously create two distinct matching reactance values within one frequency spectrum interval [18]. The multiband operation then exhibits multiband matching. Figure 1 presents the proposed network circuit topology, which is similar to a cross-architecture. The term $Z$ represents the self-impedance of each element part in the network. A cross-network allows a set of $Z_{3}, Z_{4}, \ldots$ shunt branches to extend into a cross-shaped multiband matching network architecture. Because the various shunts of $Z$ separately control distinct reactance values, the matching multifrequencies can be separately and simultaneously controlled:

$$
\begin{gathered}
Z_{\text {in }}^{*}=\left(\left(Z_{\text {Ant }}+Z_{2}\right)^{-1}+Z_{\text {Parallel }}^{-1}\right)^{-1}+Z_{1}, \\
Z_{\text {Parallel }}=Z_{3} / / Z_{4} / / Z_{5} .
\end{gathered}
$$

During matching, (1) expresses the corresponding impedance transformation in a cross-type network between the source and load. The term $Z_{\text {Parallel }}$ represents the impedance of the shunt parallel part, as expressed in (2). The two impedance values of $Z_{1}$ and $Z_{2}$ with the same capacitance value $\left(Z_{1}=Z_{2}\right), C_{1}=C_{2}$ in Figure 1 , serve as two capacitorreactance components in series to simplify the analysis. These series capacitances can also determine the matching band quality factor.

In a traditional $C$ (capacitance)- $L$ (inductance)-C(capacitance) T-type network, the load impedance of a wide-band antenna can be used to approximate the system impedance, leading to $Z_{\text {ANTENNA }} \approx Z_{0}(=50 \Omega)$, and, with the two same series capacitances $C$ and one shunt inductance $L$, the matching single operating frequency is as follows:

$$
L=\frac{1}{2}\left(C Z_{O}^{2}+\frac{1}{\left(\omega_{\text {taget }}^{2} C\right)}\right) \text {. }
$$

The theory and numerical analysis of complete impedance matching are firmly established [17]. Cross-type networks can be designed using a simple T-type network with the desired $Q$ value. A graphical method of tracing the impedance trajectory has been proposed to achieve the selectivity [17]. The selectivity in the proposed architecture can be determined by preselecting the $C_{1}$ and $C_{2}$ values. The bandwidth can be estimated using circuit $Q$ values. In dualband operation, the $C-L-C$ cross-type network simultaneously creates two matching impedance values by introducing a second branch. A tunable cross-type network uses the dual shunt of branch stubs which corresponds to the tunable match of the dual operation bands. The network design also allows simultaneous band selection and sheltering, both of which are based on the impedance-matching theorem.

The number of operation bands can be dependent on the shunt matching parts of the network. To implement a crosstype network with triple bands, each part of the network is constructed as indicated in Figure 2. In this study, an UWB [19] antenna was employed as the matching target. The antenna input impedance represents the load conditions for the numerical analysis of the cross-type network. The original broadband bandwidth of the antenna can serve as the maximal tunable range. The reflection coefficient of the original operating band affects the $S_{11}$ results; the tunable network reshapes the target band.

Based on the proposed network (Figure 2), this section introduces the element selected for each $Z$-term topology in the three operation frequencies, $f_{H}, f_{M}$, and $f_{L}$. The variable inductance value can be implemented by combining a series varactor diode with a fixed inductor in series. For a shunt branch in a triple-band network of $Z_{3}, Z_{4}$, and $Z_{5}, Z_{3}$ to the control of the high-frequency operating band $f_{H}$ branch is represented by the shunt branch $C_{6}-L_{7}-C_{8}$. The term $C_{8}$ is the variable capacitance for controlling the 


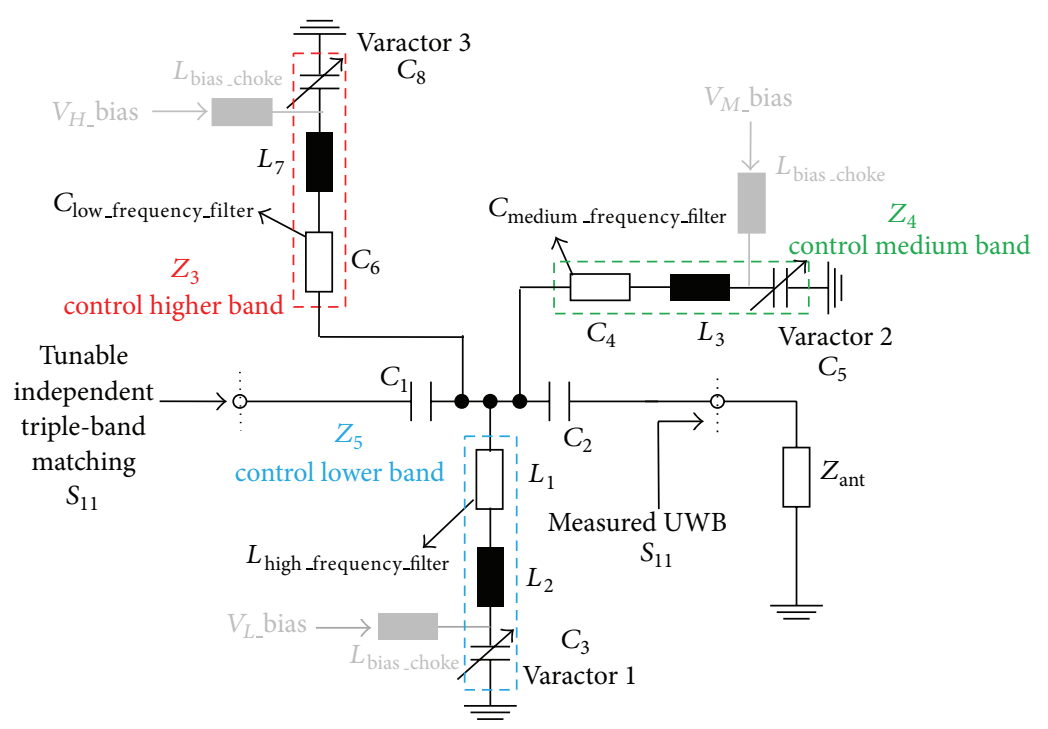

FIGURE 2: Proposed multiband network topology for triple-band.

variable inductance of the shunt branch. The placement of $C_{6}$ enables the low-frequency barrier component to isolate low-frequency signals; $Z_{5}$ to the control of the low-frequency operating band $f_{L}$ branch is represented by the shunt branch $L_{1}-L_{2}-C_{3}$. The term $C_{3}$ is also a varactor for controlling the variable inductance of the shunt branch. The placement of $L_{1}$ enables high-frequency barrier components to isolate high-frequency signals. The terms $L_{2}$ and $L_{3}$ control the inductance of the matching low- and high-frequency operating bands. Exerting $V_{L, \text { Bias }}$ on varactor $C_{3}$ to control $f_{L}$ and exerting $V_{H \text {,Bias }}$ on varactor $C_{8}$ to control $f_{H}$ enable independent adjustment of the two operating bands. The term $Z_{4}$ to the control of the middle-frequency operating band $f_{M}$ branch is represented by the shunt branch $C_{4}-L_{3^{-}}$ $C_{5}$. The function of each element is the same as previously described, except that of middle-frequency operation.

2.1. Tunable Dual-Band Operation. The networks were integrated with a broadband antenna to simulate the frequency matching and the dual-band tunable performance of antennas. In dual-band operation, the network is connected with $Z_{3}$ and $Z_{5}$ two shunt branches, as indicated in Figure 2. The capacitance and inductance devices in the network were assumed to have ideal and no-loss conditions, such that $C_{1}=$ $C_{2}=0.2 \mathrm{pF}, L_{1}=1 \mathrm{nH}, L_{2}=20 \mathrm{nH}, C_{6}=0.035 \mathrm{pF}$, and $L_{7}=15 \mathrm{nH}$. Two adjustable capacitive components, $C_{8}$ $\left(C_{H}\right)$ and $C_{3}\left(C_{L}\right)$, were configured for two distinct control terminals, where $C_{H}$ and $C_{L}$ correspond to high- and lowfrequency bands, respectively.

By employing the input impedance from the tested UWB antenna as the target-matching load, the changing capacitance of the matching network can be used to simulate a controllable operating dual band that includes a tunable low-frequency band, $f_{L}$, and a tunable high-frequency band, $f_{H}$. Figure 3 presents the control of $C_{H}$ and $C_{L}$ values with various capacitance values, which enables the $f_{L}$ and $f_{H}$ operation bands to independently adjust the frequency range from 1.72 to $6.09 \mathrm{GHz}(354 \%)$ and from 7.45 to $14 \mathrm{GHz}(188 \%)$, respectively. This design produces an extremely wide and independently tunable frequency band.

2.2. Tunable Triple-Band Operation. As indicated in Figure 2 , the objective values of the components used can be divided and fixed as follows: $L_{1}=1 \mathrm{nH}, L_{2}=40 \mathrm{nH}, C_{4}=0.05 \mathrm{pF}$, $L_{3}=20 \mathrm{nH}, C_{6}=0.05 \mathrm{pF}$, and $L_{7}=40 \mathrm{nH}$. However, $\mathrm{C}_{3}$ is the low-frequency band with the $f_{L}$ control component, $C_{5}$ is the middle-frequency band with the $f_{M}$ control component, and $C_{8}$ is the high-frequency band with the $f_{H}$ control component. These three components can independently control the three frequency bands. The performance results of the tunable triple-band antenna are presented in Figure 3, which indicates that only the values of the control capacitive component need to be adjusted to achieve independent control of the related multibands. The tuning ranges of $f_{L}, f_{M}$, and $f_{L}$ are $1.35-5.69 \mathrm{GHz}(421 \%)\left(C_{3}\right.$ tuning from 2 to $0.02 \mathrm{pF}), 3.96-8.82 \mathrm{GHz}(223 \%)\left(C_{5}\right.$ tuning from 0.5 to $0.01 \mathrm{pF}$ ), and $5.75-12.54 \mathrm{GHz}(218 \%)$ ( $C_{8}$ tuning from 0.3 to $0.01 \mathrm{pF}$ ), respectively (Figure 4). An extension of this architecture generates additional operation bands by joining additional shunt branches to the network. A back-end network system is incorporated to create multiple matching impedances, which turn the antenna into a tunable multiband antenna. The network circuit architecture can be extended to the multiband function type through this approach to increase the number of shunt matches.

\section{Analysis of a Tunable Cross-Type Network}

A theoretical equation model is presented to analyze the effectiveness of a dual-band operation network and the performance of $S_{11}$ in real operation conditions based on the two limiting factors: network structural limitations and component losses. For simplification, the analysis is conducted using dual-band $\left(f_{H}\right.$ and $\left.f_{L}\right)$ matching as an example. 


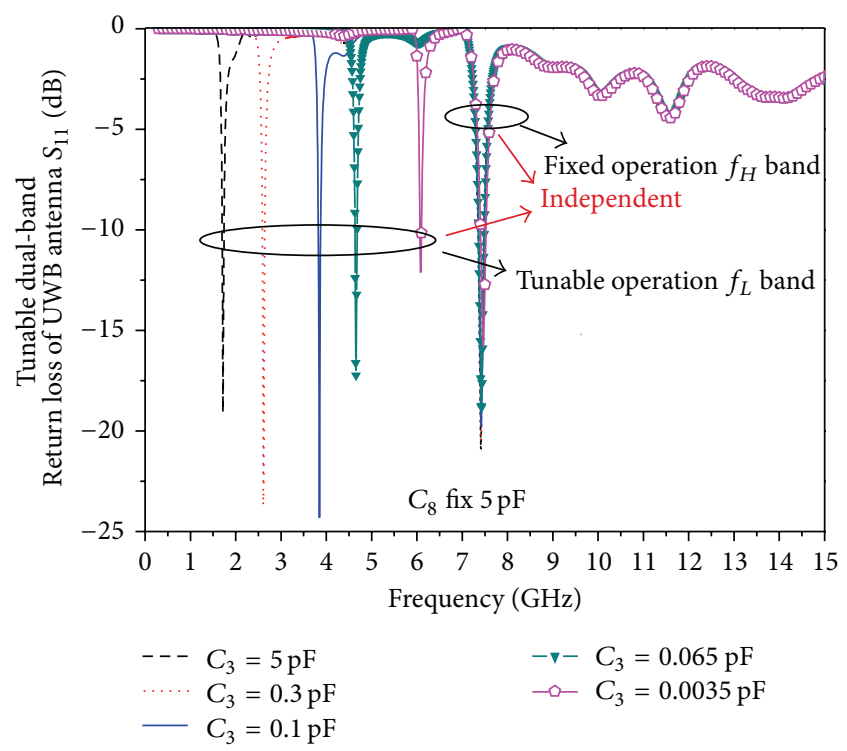

(a)

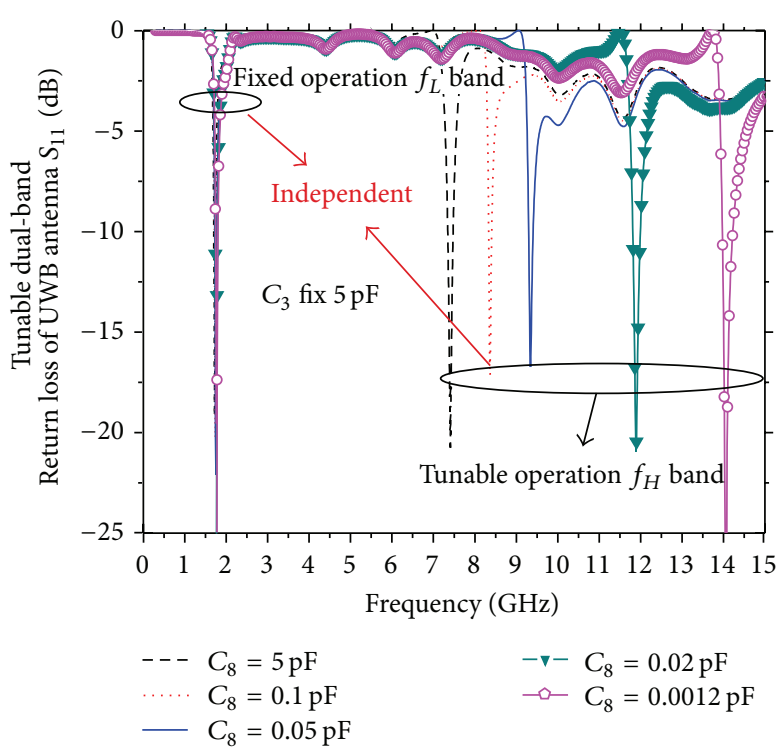

(b)

Figure 3: Simulation of independently tunable dual-band antennas at (a) $f_{L}$, (b) $f_{H}$.

3.1. Matching Operation Band Performance. Equation (2) expresses the overall shunt impedance of $Z_{\text {parallel }}$. Because the required matching reactance of dual-frequency matching is the same as that for single-frequency matching, both can be expressed by (3) under lossless conditions. The condition $C_{1}=C_{2}=0.56 \mathrm{pF}$ by (3) obtains the required reactance values for the desired dual-matching frequencies, which are two distinct inductive values. The required reactance provided by $Z_{\text {Parallel }}\left(L_{1}=0.2 \mathrm{nH}, L_{2}=6.8 \mathrm{nH}, C_{6}=\right.$ $0.47 \mathrm{pF}$, and $\left.L_{7}=3.9 \mathrm{nH}\right)$ is also considered. The two curves plotted in accordance with (2) and (3) are presented in Figure 5. The two curves in (3) and (2) that intersect at two points represent the tunable low-band $f_{L}$ and the high-band $f_{H}$ points, respectively. The interconnecting point of the $Z_{\text {parallel }}$ curve with matching reactance indicates that when $Z_{\text {parallel }}$ is between two distinct intervals bands the matching inductance value appears at the matching point. This determines whether the matching condition can occur simultaneously at dual frequencies and also the matching performance at these dual-band networks.

In Figure 5, where $C_{1}=C_{2}$, the capacitance value of $C_{8}$ is fixed at $5 \mathrm{pF}$ to achieve a fixed high-frequency $f_{H}$. Adjusting $\mathrm{C}_{3}$ from 0.5 to $5 \mathrm{pF}$ illustrates the limitations placed on the dual band by the two parallel matching architectures (Figure 5). To achieve perfect dual-band matching, the reactance function curve must match the inductance obtained using (3) to match the frequency function curve at the two intersecting points. However, within the partial band interval, when the corresponding values of $C_{3}(5 \mathrm{pF})$ decrease, the inductive range cannot intersect the matching curve, in which case the matching conditions deteriorate. When $C_{3}(0.5 \mathrm{pF})$ decreases, full capacitance appears with no matching effect, implying that the lower frequency-matching band has disappeared.
Dual matching is affected not only by $C_{3}$, but also by the relative relationship between $C_{3}$ and $C_{8}$, as (2) indicates. When two operating frequency bands are close to each other and the ratio of $C_{3}$ to $C_{8}$ is small, the inductive reactance of the low-frequency portion drops and the dual-matching results deteriorate.

3.2. Optimization Architecture for Lossy Devices. Considering the practical component loss, an investigation of the relationship between loss $Z_{\text {Parallel }}$ and the slightly dissimilar capacitance of $Z_{1}$ and $Z_{2}$, which affects matching performance, is required. The design of the cross-type dual-band network requires SMD components. The shunt branch equivalent series resistances (ESR), $r_{1}$ and $r_{3}$, represent the losses occurring at the junctions in the branch components. Because of asymmetry, it is assumed that $r_{1}=r_{3}=r$ to explain and compute the effects of associated losses, where $r$ is the total equivalent resistance of shunt branches $Z_{3}$ and $Z_{5}$. The previous derivation must also incorporate these resistance terms into the overall shunt branch $Z_{\text {Parallel_lossy }}$ as follows:

$$
\begin{aligned}
Z_{\text {Parallel_ossy }}= & Z_{3} / / Z_{5} \\
= & \left(\left(r_{1}+j \omega L_{1}+j \omega L_{2}+\frac{1}{j \omega C_{3}}\right)^{-1}\right. \\
& \left.+\left(r_{3}+\frac{1}{j \omega C_{6}}+j \omega L_{7}+\frac{1}{j \omega C_{8}}\right)^{-1}\right) .
\end{aligned}
$$

Under loss conditions, the original matching analysis is no longer applicable. A cross-type matching network for $C_{1}=$ $C_{2}$ can only be matched perfectly under an unrealistic lossless condition, and this must be considered in calculations. 


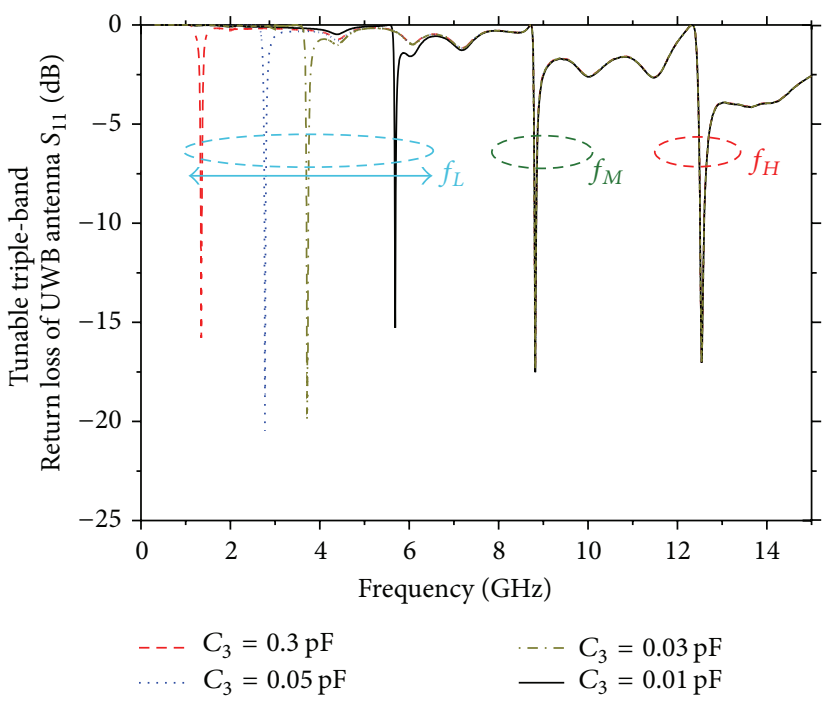

(a)

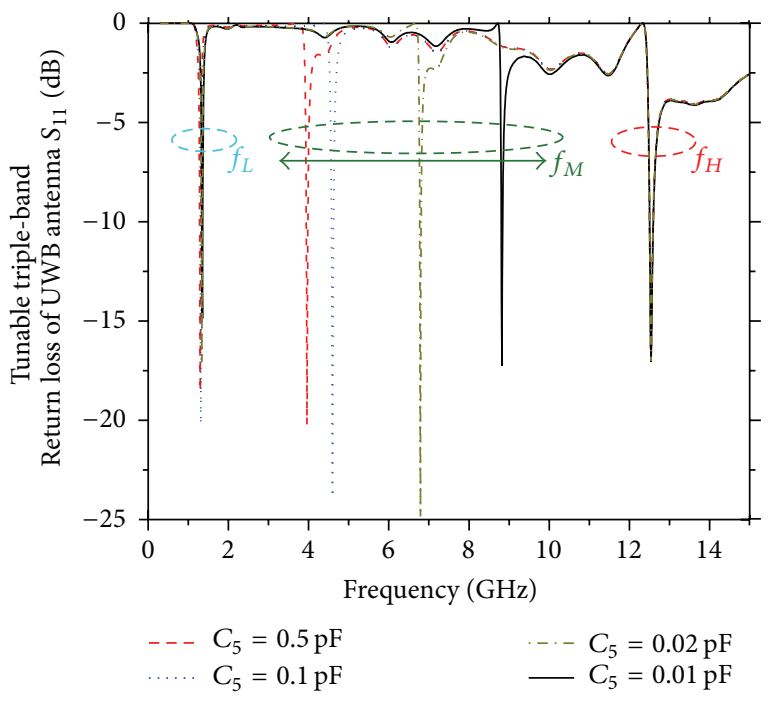

(b)

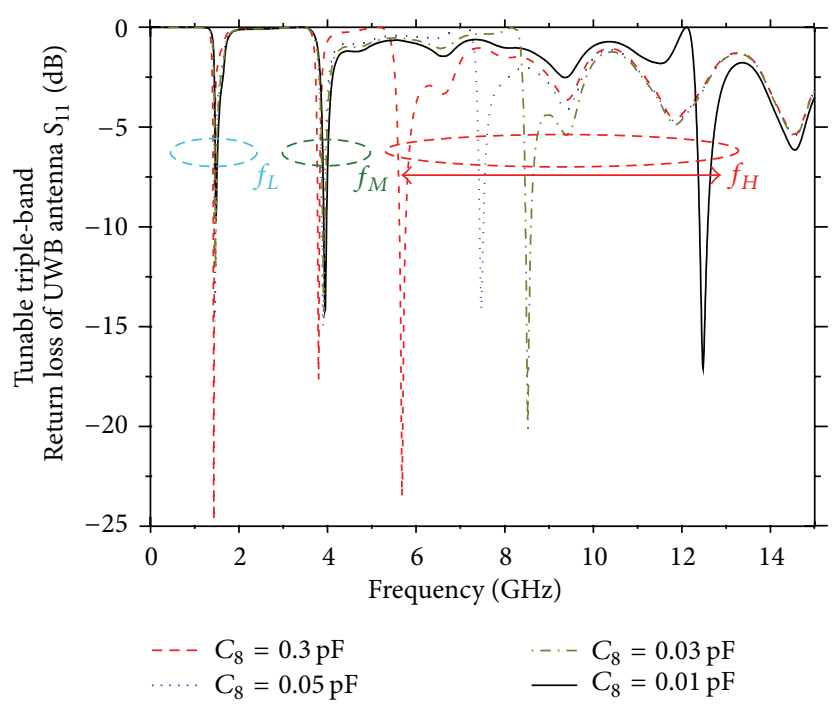

(c)

FIGURE 4: Independently continuous tunable multiband antennas at (a) $f_{L}$, (b) $f_{M}$, and (c) $f_{H}$.

Consider $Z_{\text {Parallel_lossy }}$ with loss resistance $r$, by (4) and (1), to determine the relationships among system impedance, loss resistance, and the series capacitance value for the matching frequency as follows:

$$
Z_{O}+\frac{r}{Z_{O}}-r=\frac{C_{1}}{C_{2}}
$$

This calculation indicates that it is necessary to select $C_{1}$ and $C_{2}$ by (5) to ensure appropriate matching conditions. Therefore, the network has improved insertion loss and transmission coefficient $S_{21}$. In this study, the situation with 0402 SMD capacitance and inductance components with an equivalent loss of $4.3 \Omega$ at $V_{\text {bias }}=0 \mathrm{~V}$ bias in the proposed dual-band network topology was simulated (Figure 2). As indicated in Figure 6, $S_{11}$ improved by approximately $10 \mathrm{~dB}$ by setting $C_{1}$ to $0.68 \mathrm{pF}$ and $C_{2}$ to $0.43 \mathrm{pF}$. This explains why $C_{1}$ is slightly larger than $C_{2}$ in the practical fabricated dual-band cross-type network. This design improves $S_{21}$ by approximately $1 \mathrm{~dB}$, indicating that appropriate low-loss components and a careful fabrication process can reduce insertion loss.

3.3. Group Delay of Cross-Type Network. Figure 7 shows the simulated two-port $S_{21}$ group delay of the proposed crosstype network. The simulation results indicate that the group delay is near constant over a wide range of bands for two narrow matching bands, 1.8 and $3.5 \mathrm{GHz}$. Quasi-linear group delay except form nonmatching bands, these may originate from the mixed multiple modes or several lumped element in shunt branch. Generally, the operation band has a linear phase response. The simulation group delay implies optimal 


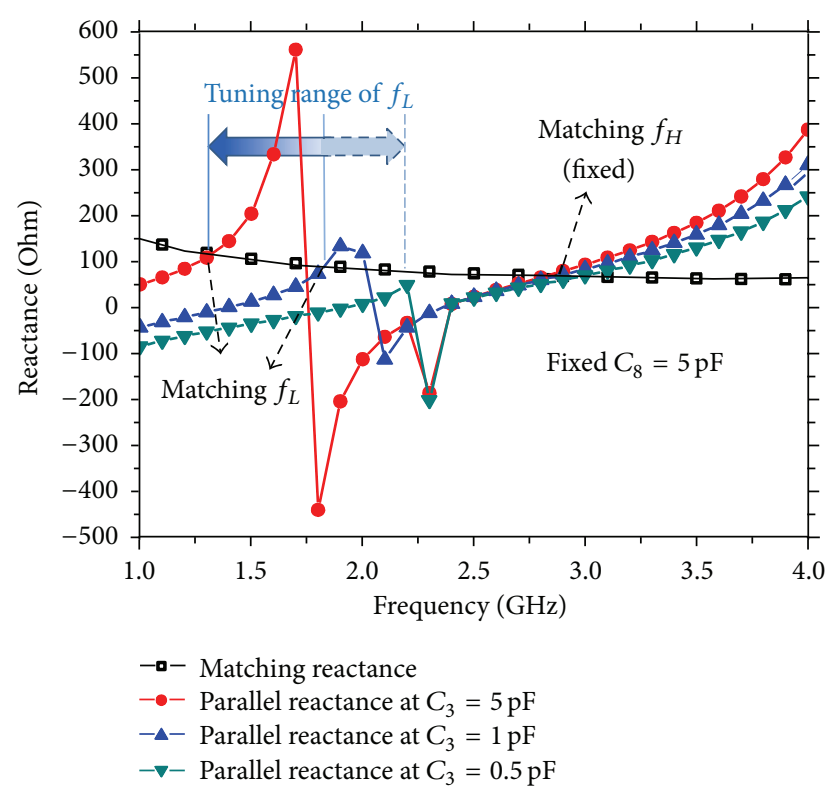

FIGURE 5: Dual-band frequency matching reactance in the crosstype network with different $C_{3}$.

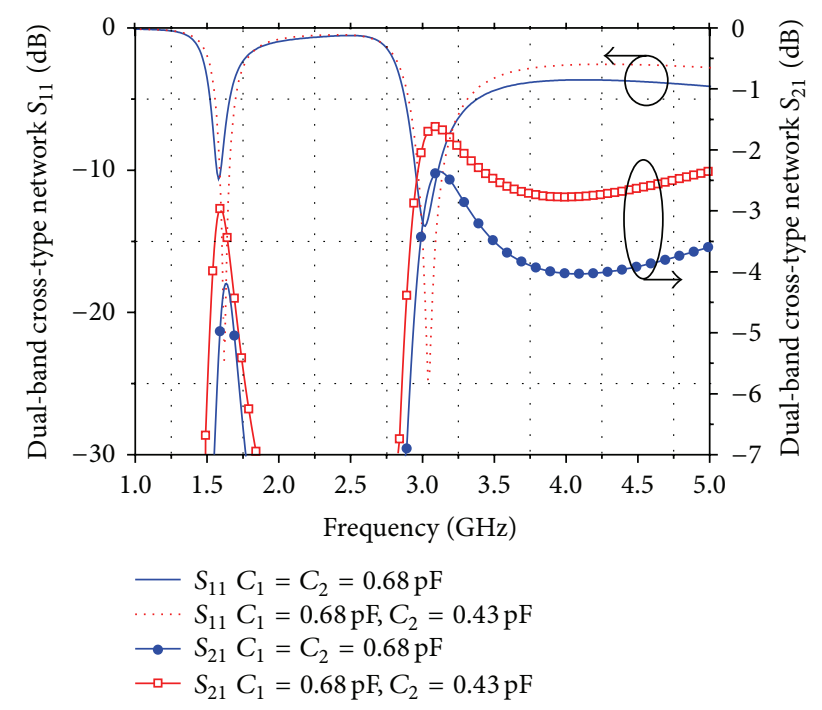

Figure 6: Lossy dual-band cross-type network with $r=4.3 \Omega$ and optimized $C_{1}$ and $C_{2}$ values.

performance of linear phase and low dispersion that ensures constant values of group delay.

\section{Fabrication and Measurement of a Tunable Cross-Type Network}

The right side of Figure 8 presents the fabricated circuit, which has a total circuit size of $2 \mathrm{~cm}^{2}$. The circuit substrate is Rogers RO4003C with thickness of $0.8182 \mathrm{~mm}, \varepsilon_{r}=$ 3.55 , and dielectric loss tangent of 0.0021 . The varactor, a GaAs hyperabrupt varactor diode (model number MGV125-25-E28) obtained from the Aeroflex/Metelics Company,

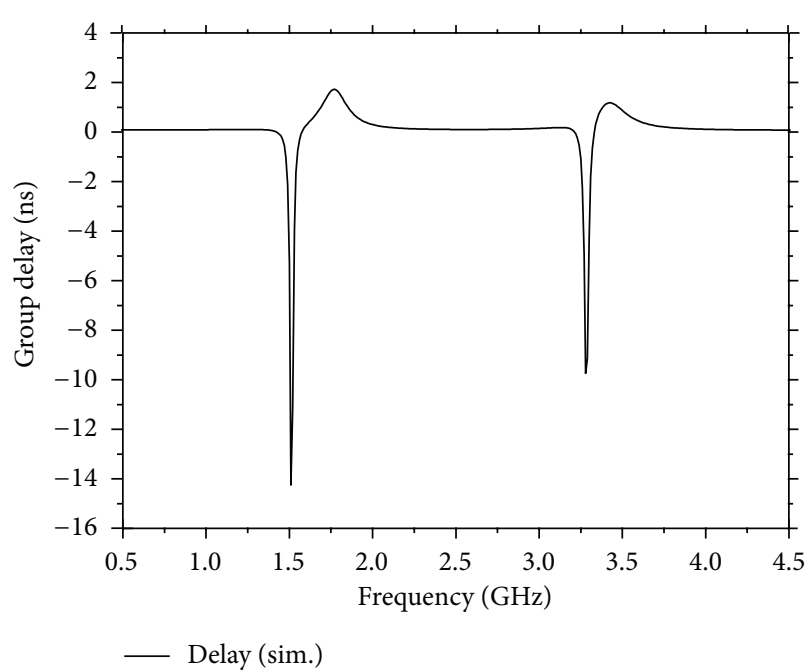

FIGURE 7: Group delay of the proposed cross-network working at $1.8 \mathrm{GHz}$ and $3.5 \mathrm{GHz}$.

continuously changes the capacitance from $0.4 \mathrm{pF}$ to $5 \mathrm{pF}$. The $Q$ value for the diode component is 3000 , and the series loss resistance is approximately $1 \Omega$ [20]. Tables 1 and 2 list the results of the cross-type network with a $50 \Omega$ load connecting the two ports, as well as the related parameters. To estimate the energy loss in this two-port network, (6) defines the transducer power gain $G_{T}$ as follows [21]:

$$
G_{T}=10 \log \frac{\left|S_{21}\right|^{2}}{1-\left|S_{11}\right|^{2}}
$$

Tables 1 and 2 indicate that the bandwidth of $f_{L}$ is relatively narrow. This is deduced from Figure 8 and explained by (2), which indicates that the reactance change in the inductance magnitude of $f_{L}$ was considerably steeper than that in the high-frequency $f_{H}$ and, therefore, has a narrower bandwidth. The network gained $G_{T}$, which is related to the loss in components and the network power transmission efficiency. The practical SMD components are lossy. Furthermore, various biases on a varactor have different $Q$ values [12] and result in varying $G_{T}$. The bias choke of the varactor is another factor affecting $G_{T}$ and the matching band selection performance. The circuit size indicated in Figure 2 can be decreased because it requires only a small area for its components. Additional transmission lines are designed to facilitate measurements.

\section{Results and Discussion of the Tunable Dual-Band Cross-Type Network Integrated with a Wideband Antenna}

The cross-type network was connected to a 1.2-4 GHz UWB antenna to create a tunable dual-band antenna. The performance of the proposed dual-band tunable antenna was verified by measuring the change in various capacitance values when various biases $(0,5,11$, and $23 \mathrm{~V})$ were applied to the first and second varactors separately. This tunable antenna can be 


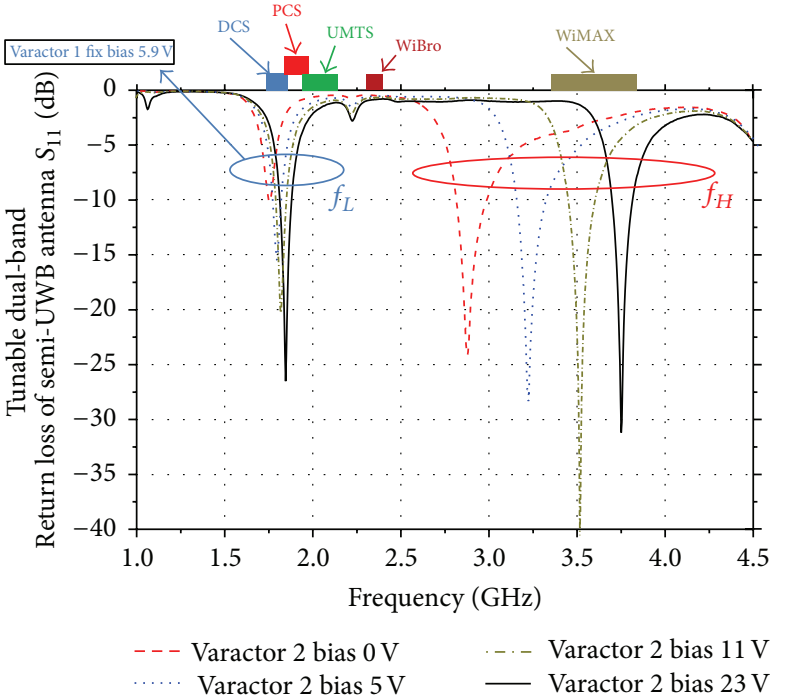

(a)

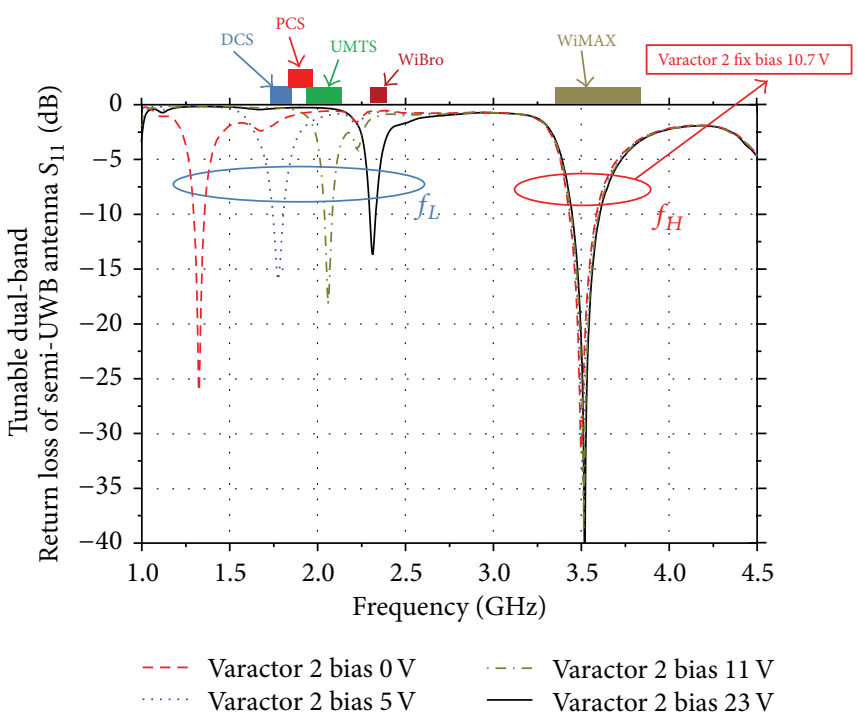

(b)

Figure 8: Measurement result of continuous tunable dual-band antenna: (a) independent tunable $f_{H}$; (b) independent tunable $f_{L}$.

TABLE 1: Performance of the tunable dual-band antenna with fixed $1.8 \mathrm{GHz}$, variable $C_{1}=0.56 \mathrm{pF}, C_{2}=0.68 \mathrm{pF}, L_{1}=0.2 \mathrm{nH}, L_{2}=6.8 \mathrm{nH}$, $C_{4}=0.47 \mathrm{pF}$, and $L_{3}=3.9 \mathrm{nH}$.

\begin{tabular}{lccccccc}
\hline$V_{\text {bias }}$ (volts) & 0 & 1 & 2 & 5 & 8 & 10 & 15 \\
Frequency $(\mathrm{GHz})$ & 2.89 & 2.96 & 3.02 & 3.22 & 3.39 & 3.48 & 3.63 \\
Return loss $(\mathrm{dB})$ & -24.1 & -24.3 & -23.1 & -24.7 & -28.9 & -32.6 & -32.8 \\
Gain $(\mathrm{dB})$ & -1.12 & -1.15 & -1.09 & -1.26 & -1.5 & -1.64 & -1.63 \\
BW $(\mathrm{MHz})$ & 144 & 156 & 156 & 168 & 156 & 156 & 132 \\
\hline
\end{tabular}

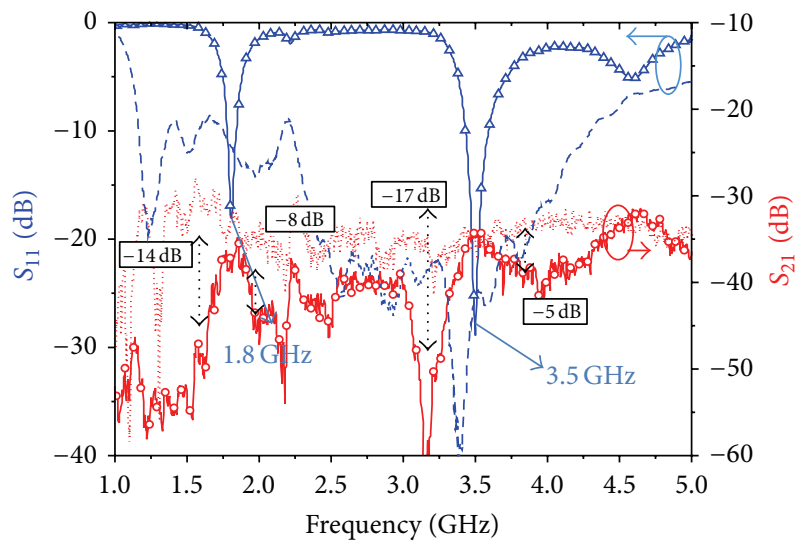

..... Original semi-UWB antenna $S_{21}$ with horn antenna

- - - Original semi-UWB antenna $S_{11}$

-o- After tunable matching $S_{21}$ with horn antenna

$\triangle-$ After tunable matching semi-UWB antenna $S_{11}$

FIGURE 9: Wireless measurement result $S_{21}$ between a tunable dualband antenna and a horn antenna. The triangle marked line indicates the tunable $S_{11}$ and the dotted line is $S_{11}$ of the original broadband antenna. The circular mark line indicates the $S_{21}$ of the proposed tunable antenna and the dashed line is $S_{21}$ of the original broadband antenna. used for multiple communication standards. Using the results of the measurements presented in Figure 8(a) as an example, the low-frequency $f_{L}$ band can be fixed in the DCS $1.8 \mathrm{GHz}$ band, whereas the other $f_{H}$ band can be independently controlled to adjust to the WiMAX band. Conversely, Figure 8 (b) indicates that the high-frequency band $f_{H}$ is fixed for WiMAX, allowing $f_{L}$ to be individually controlled and applications in other frequency bands to be adjusted. The ranges for the tunable antenna bands $f_{L}$ and $f_{H}$ are 1.39 to $2.34 \mathrm{GHz}(168 \%)$ and 2.83 to $3.72 \mathrm{GHz}(132 \%)$, and the average bandwidths of $f_{L}$ and $f_{H}$ are $51 \mathrm{MHz}$ and $148 \mathrm{MHz}$, respectively. Other frequency bands can be controlled by large variations in capacitance value after analytic calculation, allowing the antenna to have a wide tuning range. Multiple frequency bands can be expanded and adjusted similarly.

The cross-type network transferred the frequency dependence of $Z_{\text {ant }}$ to result in variation of $S_{11}$, as indicated in Figure 9. After the independent adjustment of $S_{11}$ by the dual-band cross-type network has been verified, the wireless transmission performance between antennas must also be verified by measuring $S_{21}$. A horn antenna was used at a distance of $60 \mathrm{~cm}$ (far field) to compare the effects of $S_{21}$ in changing dual-frequency operations with and without the proposed matching network. Figure 9 presents the operating 


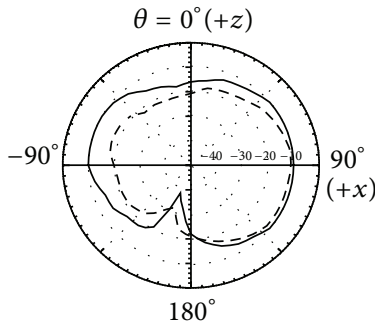

$X-Z$ plane $E_{\theta}$

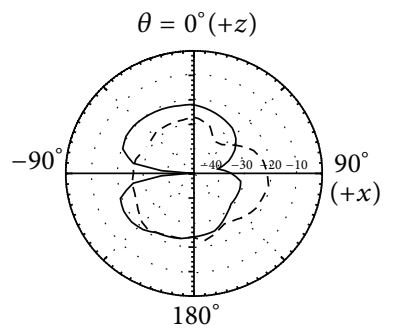

$Y-Z$ plane $E_{\theta}$
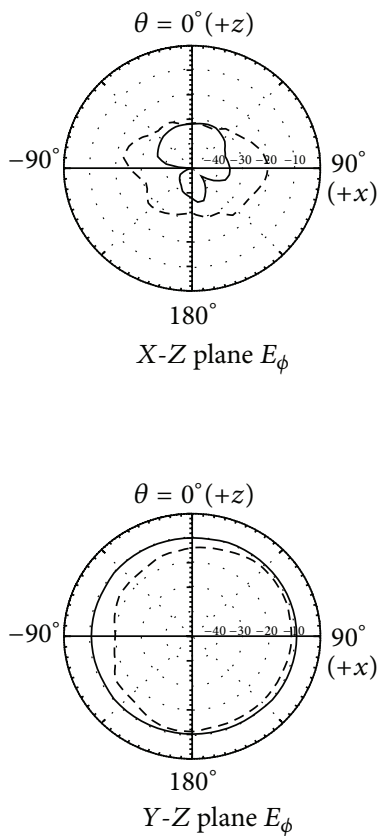

$\begin{array}{ll}\text { - } & \text { Eye-shaped antenna } \\ --- & \text { With cross-type network }\end{array}$

(a)

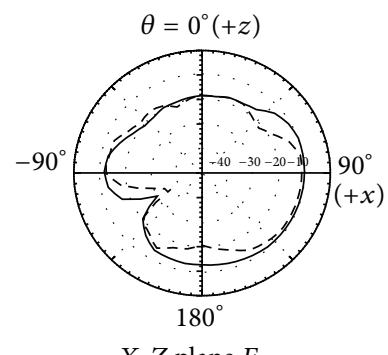

$X-Z$ plane $E_{\theta}$

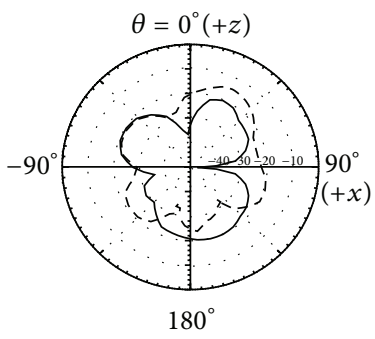

$Y-Z$ plane $E_{\theta}$

- Eye-shaped antenna

--- With cross-type network
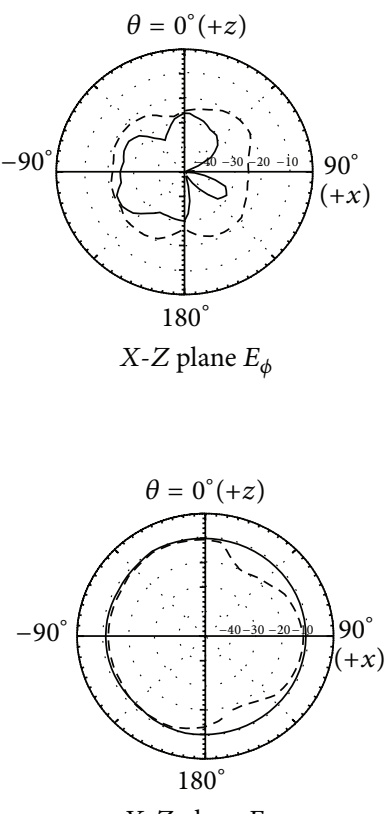

$Y-Z$ plane $E_{\phi}$

FIGURE 10: Measured 2-D radiation patterns of the tunable dual-band antenna with cross-type network and without cross-type network (eye-shaped antenna case): (a) 1.8 and (b) $3.5 \mathrm{GHz}$.

TABLE 2: Performance of the tunable dual-band antenna with fixed $3.5 \mathrm{GHz}$, variable $C_{1}=0.56 \mathrm{pF}, C_{2}=0.68 \mathrm{pF}, L_{1}=0.2 \mathrm{nH}, L_{2}=6.8 \mathrm{nH}$, $C_{4}=0.47 \mathrm{pF}$, and $L_{3}=3.9 \mathrm{nH}$.

\begin{tabular}{|c|c|c|c|c|c|c|c|c|}
\hline$\overline{V_{\text {bias }} \text { (volts) }}$ & 0 & 1 & 2 & 5 & 8 & 10 & 18 & 23 \\
\hline Frequency $(\mathrm{GHz})$ & 1.32 & 1.39 & 1.46 & 1.76 & 1.93 & 2.02 & 2.26 & 2.30 \\
\hline Return loss (dB) & -25.2 & -22.1 & -17.3 & -14.6 & -21.3 & -20.0 & -12.3 & -13.0 \\
\hline Gain (dB) & -1.38 & -1.23 & -0.81 & -0.59 & -1.96 & -2.02 & -1.18 & -1.68 \\
\hline BW $(\mathrm{MHz})$ & 60 & 60 & 50 & 72 & 56 & 48 & 36 & 36 \\
\hline
\end{tabular}

condition and wireless $S_{21}$ measurement results of the UWB antenna and this dual-band tunable matching network when the dual-band $f_{H}$ is tuned to $3.5 \mathrm{GHz}\left(V_{H \text {,bias }}=10.5 \mathrm{~V}\right)$ and $f_{L}$ to $1.8 \mathrm{GHz}\left(V_{L, \text { Bias }}=5.6 \mathrm{~V}\right)$.

At identical source signal strengths, the transmission signal in the network is highly frequency-selective compared with the original UWB antenna $S_{21}$; therefore, the signal-tonoise ratio (SNR) can be improved because of reduced noise and interference. Figure 9 presents two operated communication bands at $3.5 \mathrm{GHz}$ (data-link) and $1.8 \mathrm{GHz}$ (voice-link) simultaneously. Compared with the original broadband characteristics, the experiment indicated that the selectivity of this dual-band antenna increased substantially and demonstrated an excellent adjacent signal suppression capability of -14 to $-8 \mathrm{~dB}$ for $f_{L}$ and of -17 to $-5 \mathrm{~dB}$ for $f_{H}$, thereby enhancing the selectivity of the main signal and enabling higher-quality communication transmission. This dual-band antenna can transmit signals to other antennas effectively at an improved SNR. Such cross-type dual-band matching in practical implementation and design has already been optimized for matching network components, the insertion loss of which is approximately $-2.1 \mathrm{~dB}$ and $-2.6 \mathrm{~dB}$ for individual frequency bands $f_{L}$ and $f_{H}$ in the network, respectively.

Figure 10 presents the measured radiation patterns of the cross-type network integrated with the UWB antenna. Figure 8 indicates that the tunable antenna exhibits a nearly omnidirectional radiation pattern at both 1.8 and $3.5 \mathrm{GHz}$. Although the measurement results are comparable to the radiation patterns of the original UWB antenna (solid lines), the tunable antenna can still maintain the same electrical characteristics to the original antenna at these desired operational frequencies when a cross-type feeding network is included. The tunable cross-type network can be regarded as part of the antenna feeding network line to adjust the incident strength, so that the matching functionality at 1.8 and $3.5 \mathrm{GHz}$ can retain the natural electrical characteristics of the antenna, such as the current distributions in the radiator. The add-on cross-type network implies a negligible 
impact on the antenna patterns, but the frequency response network. Therefore, the antenna return loss is modified because the tunable cross-type frequency selectivity can be improved and dual-band tuning operation can be achieved. This omnidirectional pattern is verified to be suitable for most applications in general wireless environments and provides a tuning functionality for modifying the operation bands.

\section{Conclusion}

This paper proposes a cross-type network to fabricate a tunable multiband antenna. Adding an extra shunt branch to a network creates an additional independently tunable operating band. A minimal-control element reduces the problem of loss from variable components such as varactors and also simplifies the control units of the system. The operating frequency bands can be adjusted continuously and individually with an extremely wide tuning range. The measurements for the tunable range for this dual-band antenna were $1.39 \mathrm{GHz}$ to $2.34 \mathrm{GHz}(168 \%)$ for $f_{L}$ and $2.83 \mathrm{GHz}$ to $3.72 \mathrm{GHz}(132 \%)$ for $f_{H}$. The average bandwidth for $f_{L}$ and $f_{H}$ was $51 \mathrm{MHz}$ and $148 \mathrm{MHz}$, respectively. Therefore, the proposed novel cross-type network can be used successfully in tunable dual-band functions to decrease the processing time and analytical complexity of tunable-frequency devices. The radiation pattern of the tunable antenna exhibits a nearly omnidirectional characteristic. More than three bands can be easily extended with independent adjustment in tunable multiband antennas, based on a modified cross-type matching network with an increased number of shunt branches corresponding to independently controlled bands.

\section{Conflict of Interests}

The authors declare that there is no conflict of interests regarding the publication of this paper.

\section{References}

[1] K. Rawat, M. S. Hashmi, and F. M. Ghannouchi, "Dual-band RF circuits and components for multi-standard software defined radios," IEEE Circuits and Systems Magazine, vol. 12, no. 1, pp. 12-32, 2012.

[2] C. Zhang, S. Yang, H. K. Pan, A. E. Fathy, S. El-Ghazaly, and V. Nair, "Reconfigurable antenna for simultaneous multi-service wireless applications," in Proceedings of the IEEE Radio and Wireless Symposium (RWS '07), pp. 543-546, Long Beach, Calif, USA, January 2007.

[3] S. Yang, C. Zhang, H. Pan, A. Fathy, and V. Nair, "Frequencyreconfigurable antennas for multiradio wireless platforms," IEEE Microwave Magazine, vol. 10, no. 1, pp. 66-83, 2009.

[4] S. S. Yang, A. A. Kishk, and K. F. Lee, "Frequency reconfigurable U-slot microstrip patch antenna," IEEE Antennas and Wireless Propagation Letters, vol. 7, pp. 127-129, 2008.

[5] P. Bhartia and I. Bahl, "Frequency agile microstrip antennas," Microwave Journal, vol. 25, no. 10, pp. 67-70, 1982.

[6] Z. Jin and A. Mortazawi, "An L-band tunable microstrip antenna using multiple varactors," in Proceedings of the IEEE International Antennas and Propagation Symposium and USNC/ CNC/URSI North American Radio Science Meeting, pp. 524-527, June 2003.
[7] S. V. Shynu, G. Augustin, C. K. Aanandan, P. Mohanan, and K. Vasudevan, "Design of compact reconfigurable dual frequency microstrip antennas using varactor diodes," Progress in Electromagnetics Research, vol. 60, pp. 197-205, 2006.

[8] V. Nguyen, R. Bhatti, and S. Park, "A simple PIFA-based tunable internal antenna for personal communication handsets," IEEE Antennas and Wireless Propagation Letters, vol. 7, pp. 130-133, 2008.

[9] S. Oh, Y. Shin, and S. Park, "A novel PIFA type varactor tunable antenna with U-shaped slot," in Proceedings of the 7th International Symposium on Antennas, Propagation and EM Theory (ISAPE '06), pp. 1-3, October 2006.

[10] N. Behdad and K. Sarabandi, "Dual-band reconfigurable antenna with a very wide tunability range," IEEE Transactions on Antennas and Propagation, vol. 54, no. 2, pp. 409-416, 2006.

[11] H. Li, J. Xiong, Y. Yu, and S. He, "A simple compact reconfigurable slot antenna with a very wide tuning range," IEEE Transactions on Antennas and Propagation, vol. 58, no. 11, pp. 3725$3728,2010$.

[12] I. Carrasquillo-Rivera, R. A. R. Solis, and J. G. Colom-Ustáriz,, "Tunable and dual-band rectangular Slot-Ring antenna," in Proceedings of the Antennas and Propagation Society International Symposium, vol. 4, pp. 4308-4311, 2004.

[13] C. Lugo Jr. and J. Papapolymerou, "Six-state reconfigurable filter structure for antenna based systems," IEEE Transactions on Antennas and Propagation, vol. 54, no. 2, pp. 479-483, 2006.

[14] M. Zamudio, Y. Tawk, J. Costantine, J. Kim, and C. G. Christodoulou, "Integrated cognitive radio antenna using reconfigurable band pass filters," in Proceedings of the 5th European Conference on Antennas and Propagation (EUCAP '11), pp. 21082112, April 2011.

[15] J. Perruisseau-Carrier and P. Pardo-Carrera, "Recent developments on reconfigurable band-filtering antennas," in Proceedings of the 4th European Conference on Antennas and Propagation (EuCAP '10), pp. 1-4, April 2010.

[16] C. Chuang and S. Chung, "Synthesis and design of a new printed filtering antenna," IEEE Transactions on Antennas and Propagation, vol. 59, no. 3, pp. 1036-1042, 2011.

[17] C.-L. Yang and C.-S. Lee, "Matching network using a single control element for improving selectivity of antennas with low ringing in return loss," Progress in Electromagnetic Research C, vol. 26, pp. 29-42, 2012.

[18] C.-L. Yang, "Novel high selective band-tunable antennas over ultra-wide ranges using reconfigurable matching network," in Proceeding of the IEEE International Symposium on Antennas and Propagation and USNC/URSI National Radio Science Meeting (APSURSI '09), pp. 149-4, Charleston, Sc, USA, June 2009.

[19] C. Tsai and C. Yang, "Novel compact eye-shaped UWB antennas," IEEE Antennas and Wireless Propagation Letters, vol. 11, pp. 184-187, 2012.

[20] http://www.datasheetarchive.com/MGV-125-25-datasheet.html.

[21] C. Hoarau, N. Corrao, J. D. Arnould, P. Ferrari, and P. Xavier, "Complete design and measurement methodology for a tunable RF impedance-matching network," IEEE Transactions on Microwave Theory and Techniques, vol. 56, no. 11, pp. 2620-2627, 2008. 

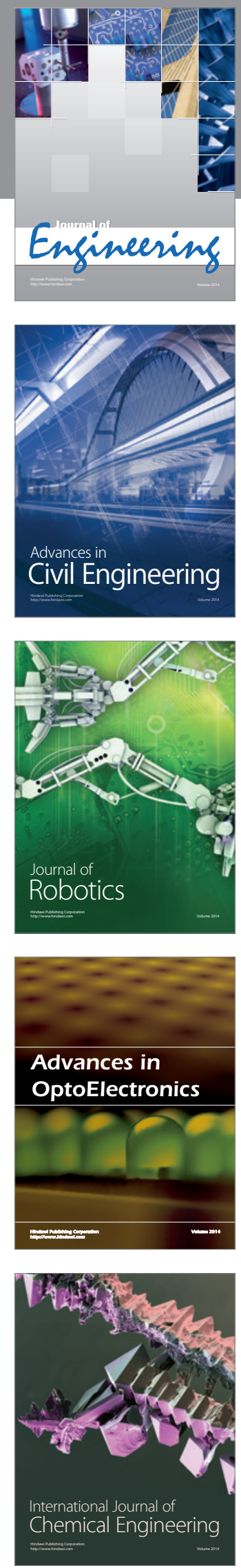

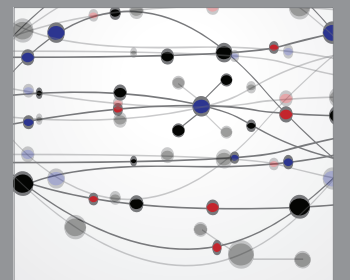

The Scientific World Journal
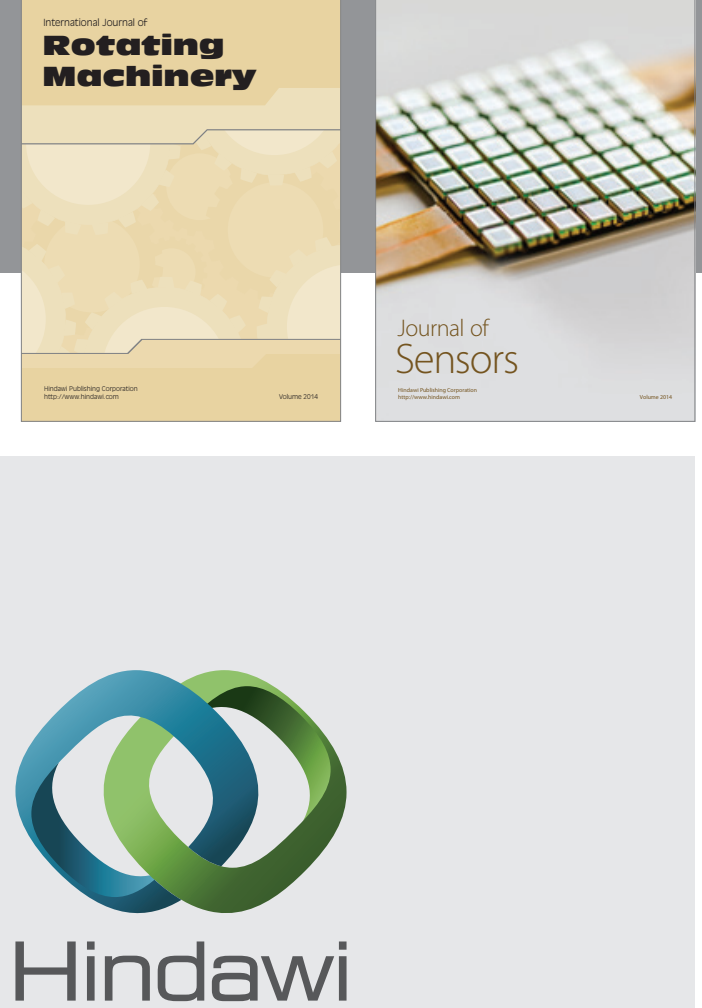

Submit your manuscripts at http://www.hindawi.com
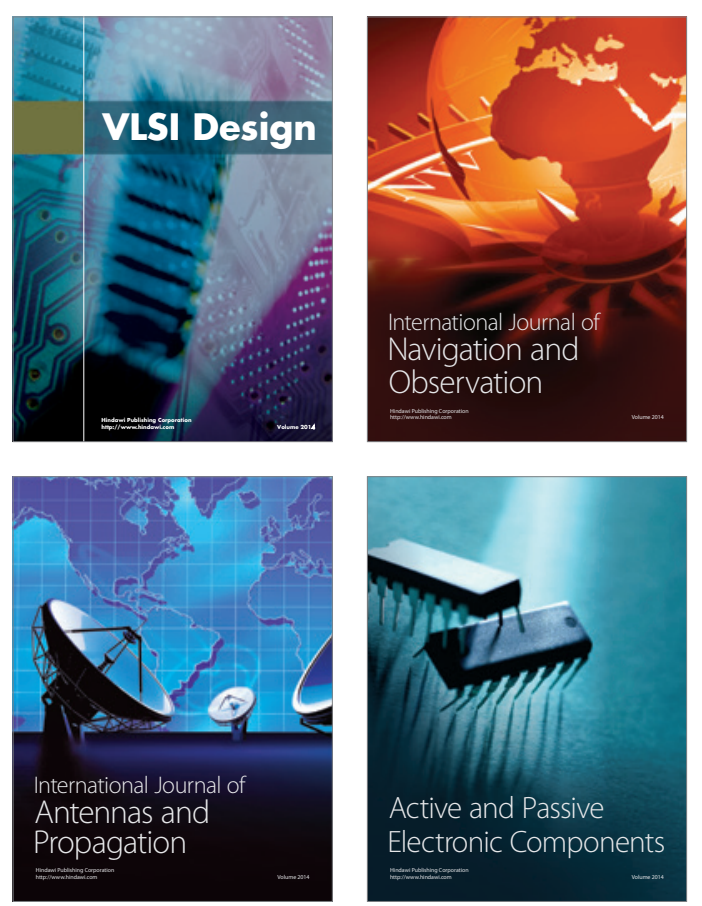
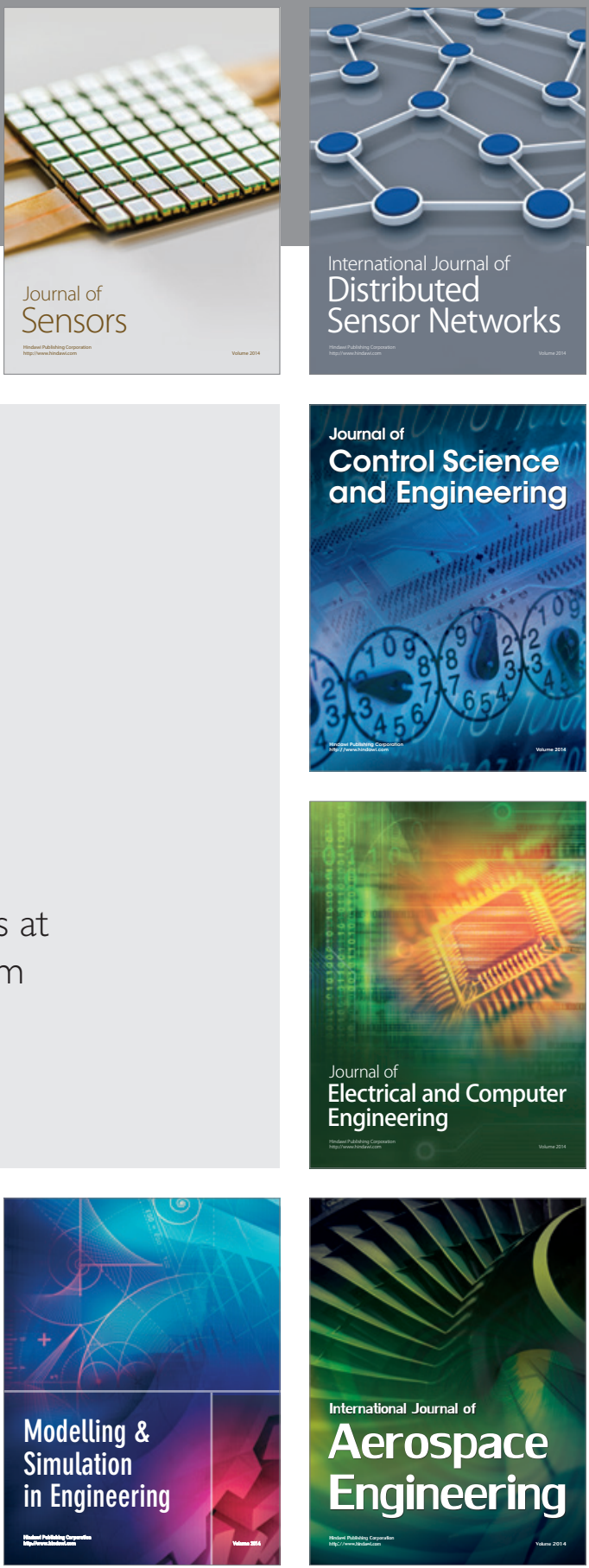

Journal of

Control Science

and Engineering
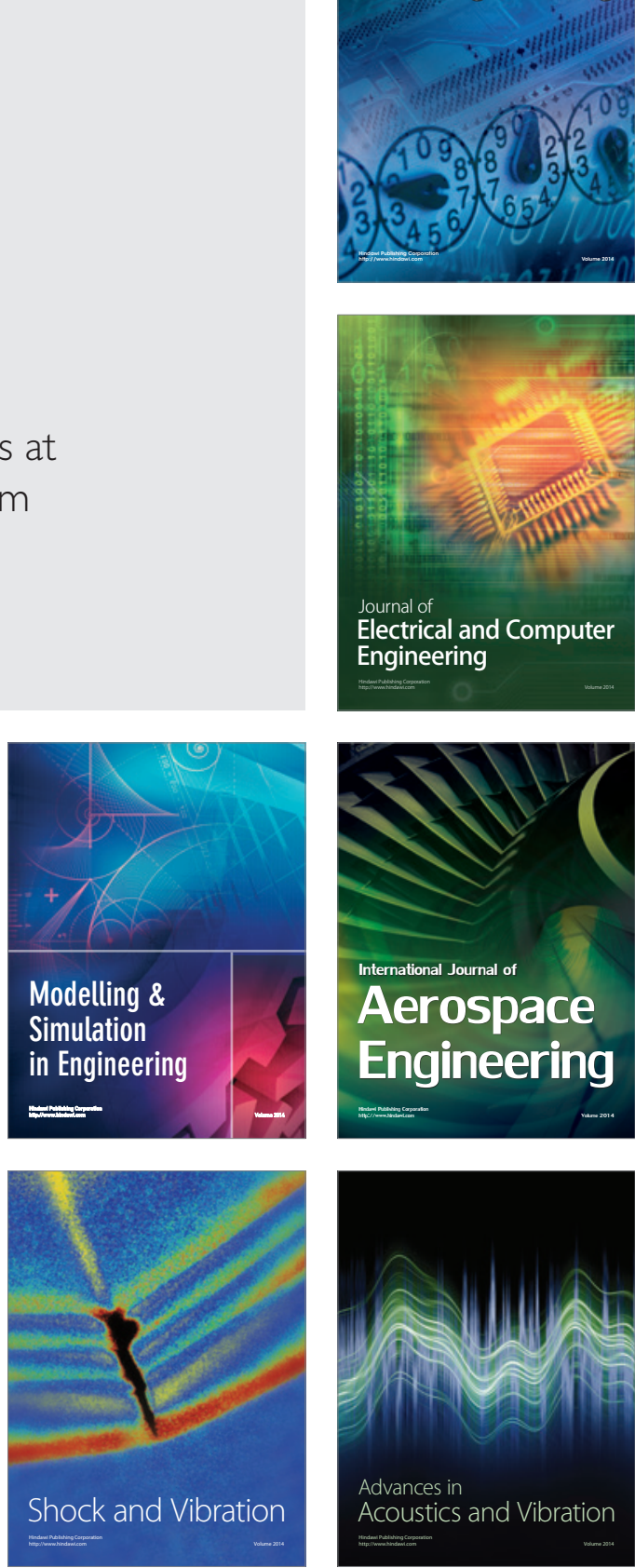